- Interventions:

- Contact nursing education about the training of collection procedures, especially with the wards that have high rejection specimen rates;

- The educational session conducted in receiving and outpatient department staff expanded to all other hospital departments;

- Collection procedure changed from four tubes to one lithium heparin tube according to insert sheet, which was a simplified procedure.

Results The quality indicator in the first 6 months showed that $0.8 \%$ of the serology samples were rejected. After the intervention using the various strategies, nurse education sessions, change of procedure, and specimen test menu information, $0.36 \%$ of the total samples were rejected, therefore showing a significant improvement in the rejection rates in serology. The continual improvement was recommended to be sustained by implementing the long-term use of the strategies used in the study.

Conclusion The project was done to improve workflow and minimize wastage in terms of time and cost, and to improve patient outcomes. This intervention was successful in the overall aims and objective of the project. The lesson learnt was that the educational session conducted as part of the intervention plan improved the skills and techniques used by nurses when performing the procedures. The altered procedure helped to significantly reduce the number of rejected specimens. The overall aim of the project was to implement a process that could be applied across all sections in the Department of Pathology and Laboratory Medicine. This was to improve patient safety, care, and outcomes.

\section{THE IMPACT OF A COMPUTERIZED PHYSICIAN ORDER ENTRY (CPOE) SYSTEM ON THE INCIDENCE AND NATURE OF DRUG-RELATED PROBLEMS (DRP) IN PEDIATRIC WARDS IN KING ABDULAZIZ MEDICAL CITY - JEDDAH}

Aeshah AlAzmi, Rph Hani AlHamdan, Omaima Ahmed, Prog Steve Tomlin, Asia Rashed. Pharmaceutical Care Services Department, King Abdulaziz Medical City Jeddah

\subsection{6/bmjoq-2019-PSF.53}

Background Prescribing a drug for a child is not a simple task because children pose distinct challenges for healthcare professionals in prescribing, dispensing, and administering any drug. Published studies investigating pediatric adverse drug events (ADEs) showed that drug ordering is the main stage of the medication process where ADEs originate and accounts for $79 \%$ of ADEs, of which $34 \%$ are related to incorrect dosing. It has been recognized that computerized physician order entry (CPOE) can reduce medication errors in adult and pediatric populations.

Methods An observational prospective cohort study was conducted on all pediatric patients aged 0-14 years admitted during the study periods to pediatric wards over a 3-month period. All reported drug-related problems (DRPs) were validated using the same method used in our previous published studies. DRPs were peer-reviewed by an expert panel consisting of a pediatrician, clinical pharmacist, and researcher. A final decision regarding validation of a DRP case was made by consensus after discussion within the group. Once a DRP was validated, the panel also assessed it for severity and preventability.

Results 657 pediatric patients were included. Of these, 235 patients suffered from 328 DRPs. Overall DRP incidence was 35.8\% (95\% CI 32.1-39.6). Almost all identified DRPs were deemed preventable $(99.7 \%)$ and $95.1 \%(n=312)$ were moderate in severity. The most frequently reported diagnoses were bronchiolitis/pneumonia $(n=32)$. Nearly half (328 [49.9\%] of 657) of patients experienced at least one DRP. The percentage of male patients with DRPs (190 [58\%] of 328) was higher than the percentage of female patients with DRPs (138 [42\%] of 328). However, there was no significant difference in DRP incidence between male and female patients $(p=0.239)$. The highest DRP incidence reported on the medical ward was $32.3 \%$ (95\% CI 27.3-37.3). The most frequently involved drug classes in DRPs were antimicrobial medications $(n=62)$, followed by respiratory medications $(n=41)$, gastrointestinal drugs $(n=21)$, vitamins $(n=14)$, steroids $(n=9)$, and nonsteroidal anti-inflammatory drugs (NSAIDs; $n=8$ ). Using the significance level of 0.05 , no significant difference was found in DRP percentages before and after CPOE use $(p=0.472)$.

Conclusion DRPs in hospitalized pediatric patients are common. The vast majority were assessed as moderate in severity and deemed preventable. In this study, the majority of DRPs reported were related to dosing and drug choice problems. Further study is needed to investigate the DRPs associated with off-label use of medication in children.

\section{EPIDEMIOLOGY OF PREVENTABLE DRUG-RELATED PROBLEMS (DRPS) IN HOSPITALIZED CHILDREN: SINGLE INSTITUTION OBSERVATIONAL STUDY}

Aeshah AlAzmi, Omaima Ahmed, Rph Hani ALHamdan, Hana AlGarni, Rawan AlZain, Rihad Althubaiti, Mohammed Aseeri, Adnan AlShaikh. Pharmaceutical Care Services Department, King Abdulaziz Medical City Jeddah

\subsection{6/bmjoq-2019-PSF.54}

Background Drug-related problems (DRPs) 'are the unwanted effects of a drug that potentially lead to harmful outcome', which receive substantial attention. Prescribing a drug for children is considered a challenging process for all healthcare providers and possesses an economic burden to the healthcare system. Hospitalized pediatric patients, in particular, represent a population at risk for DRPs. The epidemiology of preventable DRPs in children in Saudi Arabia remains scarce, which poses distinct challenges to all healthcare professionals. We aimed to characterize preventable DRPs in hospitalized children at KAMC-Jeddah.

Methods This prospective observational study included children (aged 15 years or younger) admitted to pediatric units (excluding cancer units) at King Abdulaziz Medical City (KAMC)-Jeddah, which is located in Jeddah, the largest city in Makkah Province in the Kingdom of Saudi Arabia. The hospital is a 751-bed referral center. Five diverse pediatric wards were included (general, surgical, emergency department [ED], neonatal intensive care unit [NICU], and pediatric intensive care unit [PICU]). We excluded patients admitted to pediatric cancer units, patients with no medications, if admission was less than 24 hours, and patients aged more than 15 years. The study was done over a 3-month period to determine the incidence of preventable DRPs and investigate the possible associated factors (sex, age, admission location, type of admission, and number of medications). 